\title{
Significant correlation between macroscopic and microscopic parameters for the description of localized plastic flow auto-waves in deforming alloys
}

\author{
S A Barannikova, A V Ponomareva, L B Zuev, Yu Kh Vekilov and Igor Abrikosov
}

\section{Linköping University Post Print}

N.B.: When citing this work, cite the original article.

Original Publication:

S A Barannikova, A V Ponomareva, L B Zuev, Yu Kh Vekilov and Igor Abrikosov, Significant correlation between macroscopic and microscopic parameters for the description of localized plastic flow auto-waves in deforming alloys, 2012, Solid State Communications, (152), 9, 784-787.

http://dx.doi.org/10.1016/j.ssc.2012.01.044

Copyright: Elsevier

http://www.elsevier.com/

Postprint available at: Linköping University Electronic Press

http://urn.kb.se/resolve?urn=urn:nbn:se:liu:diva-77321 

Significant correlation between macroscopic and microscopic parameters for the description of localized plastic flow auto-waves in deforming alloys

\author{
S. A. Barannikova ${ }^{1,2}$, A. V. Ponomareva ${ }^{3}$, L. B. Zuev ${ }^{1}$, Yu. Kh. Vekilov ${ }^{3}$, and \\ I. A. Abrikosov ${ }^{4}$ \\ ${ }^{1}$ Institute of Strength Physics and Materials Science, Siberian Branch of Russian \\ Academy of Science, Akademicheskii Pr. 2/4, 634021 Tomsk, Russia \\ ${ }^{2}$ Department of Physics and Engineering, Tomsk State University, 36 Lenin Prospekt, \\ 634050 Tomsk, Russia \\ ${ }^{3}$ Theoretical Physics Department, Moscow Steel and Alloys Institute, 119049 Moscow, \\ Russia \\ ${ }^{4}$ Department of Physics, Chemistry and Biology (IFM), Linköping University, SE-58183 \\ Linköping, Sweden
}

\begin{abstract}
Understanding of mechanical properties of materials and a possibility to predicting them from $a b$ initio calculations have fundamental importance for solid state theory. In this work we establish a significant correlation between the product of the macroscopic parameters of localized plastic flow auto-waves in deforming alloys, their length and propagation rate and the product of the microscopic (lattice) parameters of these materials, the spacing between close-packed planes of the lattice and the rate of transverse elastic waves. Thus, these products can be regard as invariants of plastic and elastic deformation processes, respectively. Moreover, the established regularity suggests that the elastic and the plastic processes simultaneously involved in the deformation are closely related. Our work also demonstrates that ab initio simulations can be used for the prediction of parameters of localized plastic flow auto-waves in deforming alloys.
\end{abstract}

Keywords: A. Localized plastic flow auto-waves; B. First-principles simulations; C. Metallic alloys

\title{
1. Introduction.
}

Alloying is traditionally considered as one of the most powerful methods for materials design. The physical and mechanical properties of alloys depend on their chemical content and on the internal structure which is formed during the manufacturing and service. For example, the mechanical strength and electrical resistivity are known to be very sensitive to the phase composition, microstructure, as well as to the concentrations and structure of various defects (vacancies, impurities, dislocations, grain boundaries, etc.). For alloy phases variations of the composition allows for a targeted modification of the materials characteristics. Consequently, an understanding of fundamental properties of materials, e.g. their mechanical behavior, is a challenging problem for the modern materials science. 
It is currently accepted that the deformation of a solid exerted to cyclic loading or shock excitation occurs via wave processes. However, speculation continues concerning elastic waves, which occur in an elastically deforming solid and plastic waves, which are distinct from the former in the sense that they are associated with the propagation of plastic flow fronts in the deforming material. During the recent decades numerous investigations have addressed the generation of the above two classes of waves in a deforming solid [114]. The physical properties of these waves and the conditions favoring their generation have been studied extensively. Nonetheless, recent investigations suggest that waves of yet another kind are likely to be generated in a solid subjected to tensile loading at a constant rate $[1,2]$.

In particular, the experimental studies of plastic flow in metals and alloys [1-5] have unambiguously demonstrated its tendency towards localization behavior from yield point to failure. Typical localization patterns observed by mechanical testing using a technique of double-exposure speckle-photography [6] are shown in Fig. 1. Figure 1 indicates that the patterns have very complex structure characterized by a typical macroscopic scale of about $10^{-2} \mathrm{~m}$. This suggests that the deforming medium becomes spontaneously stratified into macroscopic layers, with deforming (active) layers alternating with non-deforming (passive) ones. In a general case, the boundaries between such layers are mobile; therefore, the process of plastic flow is conventionally considered as evolution of localized plastic flow zones.

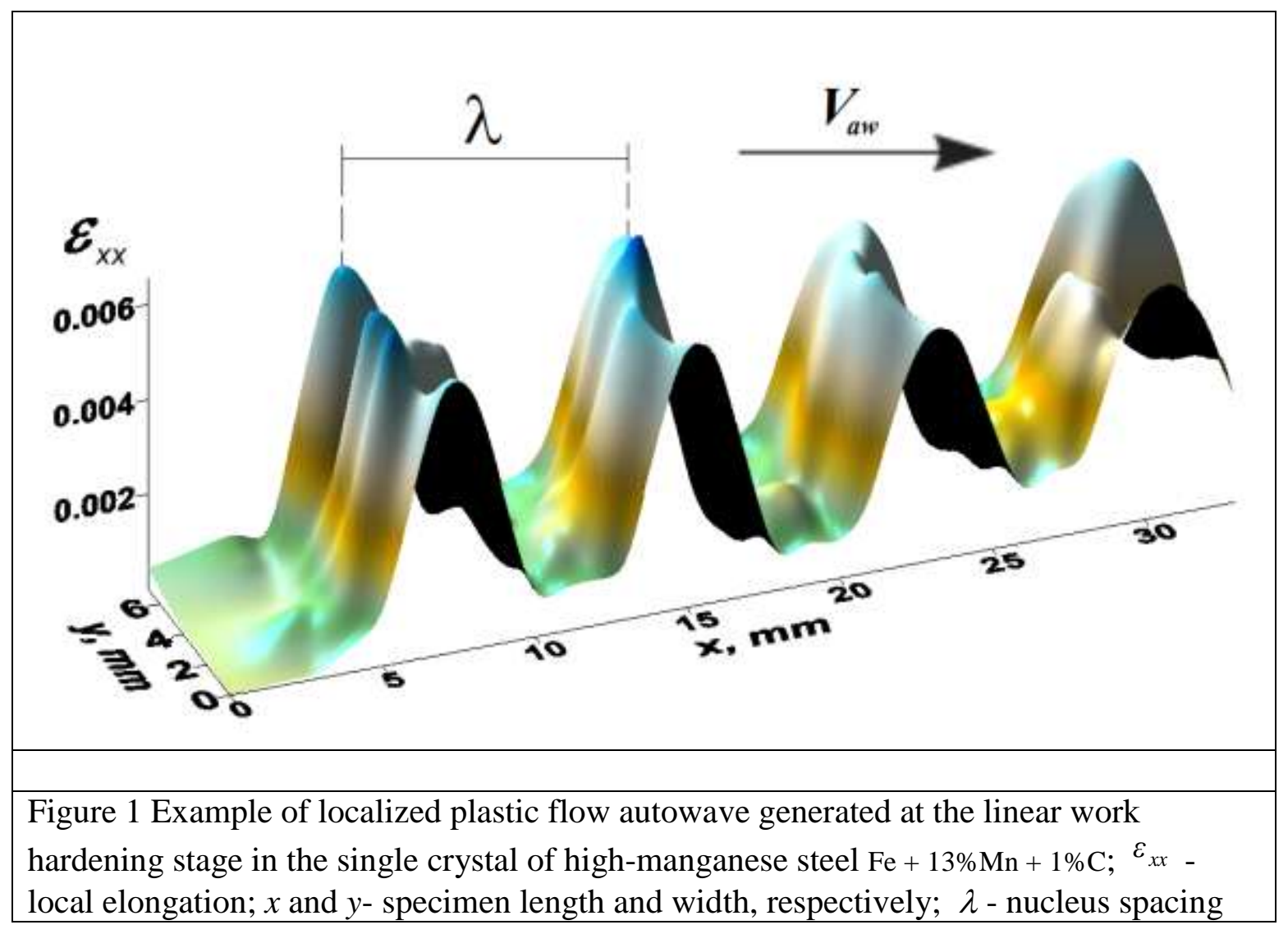


(autowave length); $V_{a w}$ - autowave propagation rate. The kinetics of macro-localization pattern evolution was investigated using time evolution of local nuclei's positions, as discussed below (see Fig. 2).

The localization phenomenon is a self-organization processes [1,2], where the term 'selforganization' is used, according to Haken [7], to indicate that the system acquires spatial, temporal or functional structure in the absence of any specific periodic external action. Importantly, the localized plastic flow patterns have all the particular features of autowave (self-excited) process. Moreover, it has been shown that the emergent localized plastic flow patterns strictly correspond to the well-known plastic strain stages [10], and the rule of correspondence has been introduced [8,9], stating that in accordance with the acting work hardening law each plastic flow stage involves a special kind of autowave process. The autowave features of the localized plastic flow patterns are of major importance at the linear stage of deformation hardening as the plastic flow localization takes on the form of phase autowave, which has length $\lambda \approx 10^{-2} \mathrm{~m}$ and propagation rate $10^{-5} \leq V_{a w} \leq 10^{-4} \mathrm{~m} \cdot \mathrm{s}^{-1}$. The autowaves in question are distinct from the well-known plastic deformation waves that are generated in solids under shock loading which are described by Kolsky in [13].

For a set of pure metals a phenomenological model of plastic flow localization introduced $[8,9]$, succeeded to correlate macroscopic parameters of the autowaves, $\lambda$ and $V_{a w}$ for easy glide and linear work hardening stages to the materials specific microscopic parameters, the spacing $d$ between close-packed planes of the lattice and is the rate of transverse elastic waves $V_{\perp}$ :

$$
\lambda V_{a w} \approx 1 / 2 d V_{\perp}
$$

In this work we establish a reliability of this model for the important case of metallic alloys. Because in this case reliable experimental information on the materials specific microscopic parameters are often not available, we determine them form first-principles computer simulations [15], which are known to give reliable results for thermodynamic and mechanical properties of metallic systems, including disordered alloys, without any adjustable parameters fitted to the experiment [16]. Using the experimentally determined autowave length and propagation rate for a set of technologically important alloys and the interplanar distance $d$ and transverse elastic waves velocity $V_{\perp}$, calculated by means of the Exact Muffin Tin Orbital (EMTO) Method combined with the Coherent Potential Approximation (CPA) [17] we extend the validity of Eq. (1) to a qualitatively new class of materials systems.

\section{Experimental determination of autowaves macroscopic parameters.}

The alloy systems consisted in the present study are summarized in Table I. All these are technologically important materials. For example, austenitic Fe-Cr-Ni alloys belong to the important class of steels, stainless steels, known for their corrosion resistance and 
excellent mechanical properties, including strength, stiffness, and toughness. Fe-Mn alloys are known for their extraordinary strengthening behavior during plastic deformation. The iron-silicon system is important for metallurgy, thermoelectric applications, electrotechnic industry, and geophysics [18,19]. Zr-Nb alloys are of high Table I. Alloy systems considered in the present study.

\begin{tabular}{|c|c|c|}
\hline Alloy composition (wt. \%) & $\begin{array}{l}\text { Crystal } \\
\text { structure }\end{array}$ & $\begin{array}{l}\text { Single crystal growth direction } \\
\text { /polycrystalline (grain size in } \mathrm{mm} \text { ) }\end{array}$ \\
\hline $\mathrm{Fe}+18 \% \mathrm{Cr}+18 \% \mathrm{Ni}+0.35 \% \mathrm{~N}$ & fcc & Single crystal [1 11], [001] \\
\hline $\mathrm{Fe}+13 \% \mathrm{Mn}+1 \% \mathrm{C}$ & fcc & 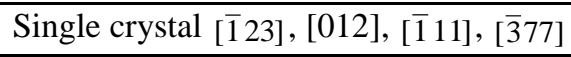 \\
\hline $\mathrm{Fe}+3 \% \mathrm{Si}$ & bcc & Single crystal [143] \\
\hline $\mathrm{V}+2,3 \% \mathrm{Zr}+0,4 \% \mathrm{C}$ & $\mathrm{bcc}$ & Polycrystalline $\left(5 \cdot 10^{-3}\right)$ \\
\hline $\mathrm{Zr}+1 \% \mathrm{Nb}$ & hcp & Polycrystalline $\left(3 \cdot 10^{-3}\right)$ \\
\hline $\mathrm{Ti}+2,8 \% \mathrm{Al}+5 \% \mathrm{Mo}+4,5 \% \mathrm{~V}$ & hcp & Polycrystalline $\left(9 \cdot 10^{-3}\right)$ \\
\hline $\mathrm{Cu}$ & fcc & Single crystal [i 39$]$ \\
\hline $\mathrm{Ni}$ & fcc & Single crystal [1] 67$]$ \\
\hline
\end{tabular}

interest for nuclear energetic. Vanadium-based alloys are considered as attractive materials for high-temperature applications. To establish a correspondence with earlier studies [8,9], we also considered pure metallic systems, fcc single crystals of $\mathrm{Cu}$ and $\mathrm{Ni}$.

All the single crystals, from which the test specimens were cut out, had been grown by the Bridgman method in the atmosphere of inert gas. The test specimens were doublespade like in shape and had thickness $1.3 \ldots 1.5 \mathrm{~mm}$; their gauge part had dimensions $28 \times 5 \mathrm{~mm}$. These were subjected to tensile loading at a constant rate at room temperature on a universal test machine "Instron-1185".

The experimental observation of localized plastic flow autowaves was carried out by means of a specially developed speckle photography technique related to focused-image holography [6]. The technique is implemented within Automatic Laser Measuring Complex (ALMEC) [6], allowing for the reconstruction of the displacement vector field during deformation of flat samples. The spatial distributions of plastic distortion tensor components can be used to locate localized plastic flow nuclei; the kinetic characteristics of the nuclei can be determined from the temporal evolution thereof. The characteristics of autowaves are determined as the distance between the localization nuclei (length of autowave, $\lambda$, Fig. 1). Considering their temporal evolution (Fig. 2), one finds the rate of autowave propagation $V_{a w}$ from the angle of the line connecting positions of the nuclei at different times. Table II summarizes experimentally measured characteristics of autowaves, $\lambda$ and $V_{a w}$ for the systems studied in this work. 


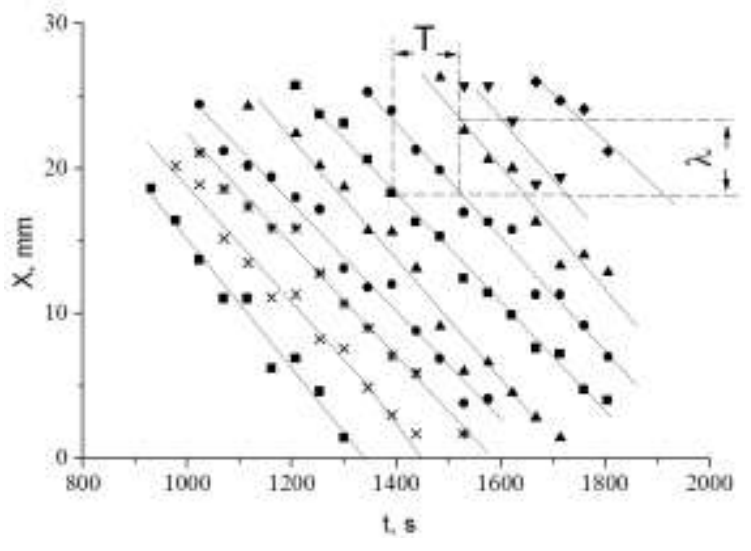

Figure 2. Procedure for measuring wavelength, $\lambda$, and oscillation period, $T$, of autovewes. Distance $X$ is measured along the specimen length, $t$ shows the loading time, points represent local nuclei's positions. The motion rate $V_{a w}=\lambda / T$. Example in the figure shows the measurements for the single crystal of high-manganese steel $\mathrm{Fe}+13 \% \mathrm{Mn}$ $+1 \% \mathrm{C}$.

Table II. Experimentally determined macroscopic parameters of the autowaves $\lambda$ nucleus spacing (autowave length); $V_{a w}$ - autowave propagation rate, calculated microscopic parameters, $\mathrm{d}$ - the spacing between close-packed planes of the lattice and the rate of transverse elastic waves $V_{\perp}$ for metals and alloys considered in this work (Table I). The corresponding products of macroscopic and microscopic parameters from Eq. (1), as well as the ratio, Eq. (2), are also presented.

\begin{tabular}{|c|c|c|c|c|c|c|c|c|}
\hline № & Alloy system $^{a}$ & $\begin{array}{c}\lambda \cdot 10^{-3} \\
\mathrm{~m} \\
\text { exp. }\end{array}$ & $\begin{array}{c}V_{a w} \cdot 10^{5} \\
\mathrm{~m} / \mathrm{s} \\
\exp .\end{array}$ & $\begin{array}{c}\lambda \cdot V_{a w} \cdot 10^{7} \\
\mathrm{~m}^{2} / \mathrm{s} \\
\text { exp. }\end{array}$ & $\begin{array}{c}V_{\perp} \cdot 10^{-3} \\
\mathrm{~m} / \mathrm{s} \\
\text { calc. }\end{array}$ & $\begin{array}{c}d \cdot 10^{10} \\
\mathrm{~m} \\
\text { calc. }\end{array}$ & $\begin{array}{c}d \cdot V_{\perp} \cdot 10^{7} \\
\mathrm{~m}^{2} / \mathrm{s} \\
\text { calc. }\end{array}$ & $\frac{2 \lambda V_{a w}}{d V_{\perp}}$ \\
\hline 1 & $\mathrm{Fe}-\mathrm{Cr}-\mathrm{Ni}$ & 5.0 & 5.1 & 2.55 & $2.39^{b}$ & $2.10^{b}$ & $5.02^{b}$ & $1.02^{b}$ \\
\hline & & & & & $2.48^{\mathrm{c}}$ & $2.08^{\mathrm{c}}$ & $5.16^{c}$ & $0.99^{c}$ \\
\hline 2 & Fe-Mn & 5.0 & 4.7 & 2.35 & $2.18^{c}$ & $2.08^{\mathrm{c}}$ & $4.53^{c}$ & $1.04^{\mathrm{c}}$ \\
\hline 3 & $\mathrm{Fe}-\mathrm{Si}$ & 4.3 & 5.2 & 2.24 & $2.52^{b}$ & $2.01^{b}$ & $5.07^{b}$ & $0.88^{b}$ \\
\hline & & & & & $2.56^{\mathrm{c}}$ & $2.00^{\mathrm{c}}$ & $5.12^{c}$ & $0.88^{c}$ \\
\hline 4 & $\mathrm{~V}-\mathrm{Zr}$ & 4.0 & 7.0 & 2.80 & 2.87 & 2.13 & 6.11 & 0.92 \\
\hline 5 & $\mathrm{Zr}-\mathrm{Nb}$ & 5.5 & 3.5 & 1.93 & 2.04 & 2.46 & 5.02 & 0.77 \\
\hline 6 & Ti-Al-Mo-V & 7.0 & 5.0 & 3.50 & 2.80 & 2.22 & 6.22 & 1.13 \\
\hline 7 & $\mathrm{Cu}$ & 4.5 & 8.0 & 3.60 & 2.18 & 2.10 & 4.58 & 1.57 \\
\hline 8 & $\mathrm{Ni}$ & 3.5 & 6.0 & 2.10 & $2.59^{b}$ & $2.04^{b}$ & $5.28^{b}$ & $0.80^{b}$ \\
\hline
\end{tabular}

${ }^{a}$ For exact compositions of the alloys and their crystal structures see Table I. The influence of interstitial impurities is not considered in calculations.

${ }^{\mathrm{b}}$ Ferromagnetic order is assumed in calculations.

${ }^{\mathrm{c}}$ Calculations are carried out within the disordered local moment model simulating the paramagnetic state above the magnetic transition temperature. 


\section{Theoretical calculations of materials specific microscopic parameters}

Materials specific microscopic parameters, the spacing $d$ between close-packed planes of the lattice and is the rate of transverse elastic waves $V_{\perp}$ were calculated from the firstprinciples using the EMTO-CPA method [17]. Its use in this work was motivated by a successful track record of this method in earlier applications [17-23]. In addition, we employed the Debye-Grüneisen theory of Ref. [24] that relates $V_{\perp}$ to share modulus $S$ and suggests to estimating $S$ from bulk modulus $B$ via relation $S \sim 0.3 B$, giving the final expression for the rate of transverse elastic waves:

$$
V_{\perp}=\sqrt{\frac{S}{\rho}} \approx \sqrt{\frac{0.3 B}{\rho}} \quad .
$$

Despite its simplicity, the approach has been shown to give reliable results for pure metals [24], as well as for alloys [25,26].

For the exchange-correlation energy and one-electron potential, following the arguments of Ref. [16], we obtain the self-consistent electron densities within the local-density approximation (LDA) and then calculate the total energies in the generalized gradient approximation (GGA) using full charge density formalism [27]. The Brillouin zone integration was carried out using 1240, 1240 and 679 k-points for the fcc, bcc, and hcp systems, respectively. The energy integration, needed to determine the charge density within the EMTO method has been carried out in the complex plane using a semi-elliptic contour comprising 24 energy points. The calculations were performed for a basis set including valence $s p d f$-orbitals, whereas the core states were recalculated at each iteration of the self-consistency loop. In our CPA calculation we included the screening contribution to the electrostatic potential and energy [16] to take into account the effect of charge transfer between the alloy components. For Fe-Si and $\mathrm{Fe}-\mathrm{Cr}-\mathrm{Ni}$ alloys, we considered ferromagnetic, as well as the so-called disordered local moment (DLM) [18] magnetic configurations, the latter being an accurate model for the simulation of the paramagnetic state above the magnetic transition temperature [18, 22, 23]. Fe-Mn alloys were simulated only within the DLM model, as it gives more accurate description of their elastic properties [29]. Because the CPA does not allow one to treat the effects of local lattice relaxations, they were neglected in the present study. For this reason, we limited theoretical simulations by substitutionally disordered alloys, and we did not considered the influence of $\mathrm{C}$ or $\mathrm{N}$ impurities on the properties of alloy systems, studied experimentally (Table 1). This is a reasonable approximation for establishing qualitative correlations between macroscopic and microscopic parameters, which is the main goal of the present work. Modified Morse equation of state [24] was used to fit energy dependences on volume for each system considered in this study.

Table II summarizes calculated values of $d$ and $V_{\perp}$ for the systems studied experimentally in this work. Note that the experimental information is available for only few systems (pure $\mathrm{Cu}$ and $\mathrm{Ni}$, as well as $\mathrm{Fe}-\mathrm{Si}$ and $\mathrm{Fe}-\mathrm{Mn}$ alloys), and accuracy of the calculated interatomic distances is found to be rather high, better than $1 \%$. A comparison between 
calculated and experimental bulk moduli shows, as expected, somewhat lower, with the error around $10 \%$. Still, this is a typical accuracy of first-principles simulations, confirming the reliability of the EMTO-CPA method, chosen for the present work. However, giving additional approximations introduced by the Debye-Grüneisen theory [20] we should expect the accuracy of calculated values for $V_{\perp}$ to be somewhat lower as compared to the interlayer spacing $d$. At the same time, the CPA, adopted for the treatment of the substitutional disorder is seen to be quite accurate, and does not introduce any additional errors to the calculated lattice parameters and bulk moduli as compared to pure elements. Thus, our theoretical tools are sufficiently accurate for the main purpose of the present work, that is to investigate the phenomenological correlation between macroscopic parameters of the autowaves, $\lambda$ and $V_{a w}$ and materials specific microscopic parameters, $d$ and $V_{\perp}$, given by Eq. (1), extending the treatment from pure elements to the alloy phases.

It is interesting to point out that magnetic effects, which often strongly influence thermodynamic properties of metallic alloys [20, 22, 23, 29], turn out to be less significant for systems considered in this study. Indeed, both interlayer spacing $d$ and $V_{\perp}$, calculated for bcc Fe-Si alloy, as well as for fcc Fe-Cr-Ni alloy turns out to be quite close to each other. At the same time, we would like to call attention to the following important fact. Fe-Cr-Ni alloys with compositions relevant for the present study have very low magnetic ordering temperature, and at room temperature they are paramagnetic [30]. As emphasized in Ref. [31], a treatment of paramagnetic system as nonmagnetic can lead to erroneous conclusion. In particular, our nonmagnetic calculations carried out for the random fcc $\mathrm{Fe}_{72} \mathrm{Cr}_{16} \mathrm{Ni}_{12}$ alloy show that within this approximation lattice parameter (3.48 $\AA$ ) and bulk modulus (264 GPa) are significantly different from the values calculated within the DLM model, $3.54 \AA$ and $156 \mathrm{GPa}$, for the lattice parameter and $B$ respectively. Thus, the adoption of the DLM description for the paramagnetic phases of magnetic alloys considered in this study is essential for their proper description.

\section{Discussion}

A comparison between calculated products of microscopic material specific parameters $d$ and $V_{\perp}$ and measured macroscopic parameters of the autowaves $\lambda$ and $V_{a w}$, obtained for studied metals and alloys is given in Table II. Also, Fig. 5 clearly demonstrates that the dependence of $\lambda / d$ ratio on $V_{\perp} / V_{a w}$ ratio is nearly linear. Matching this data suggests that the phenomenological correlation, given by Eq. (1) is fulfilled within an acceptable range of accuracy also in case of alloy systems, investigated in this work.

Moreover, Eq. (1) is validated by the fact that the average ratio $\left\langle 2 \lambda \cdot V_{a w} / d \cdot V_{\perp}\right\rangle \approx 1.01 \pm 0.2$ obtained for studied metals and alloys is quite close to unity.

Equation (1) is physically significant since it establishes a quantitative relationship between the micro-scale (lattice) characteristics $\left(d\right.$ and $V_{\perp}$ ) of elastic waves which govern elastic deformation processes on the one hand and the macro-scale characteristics 


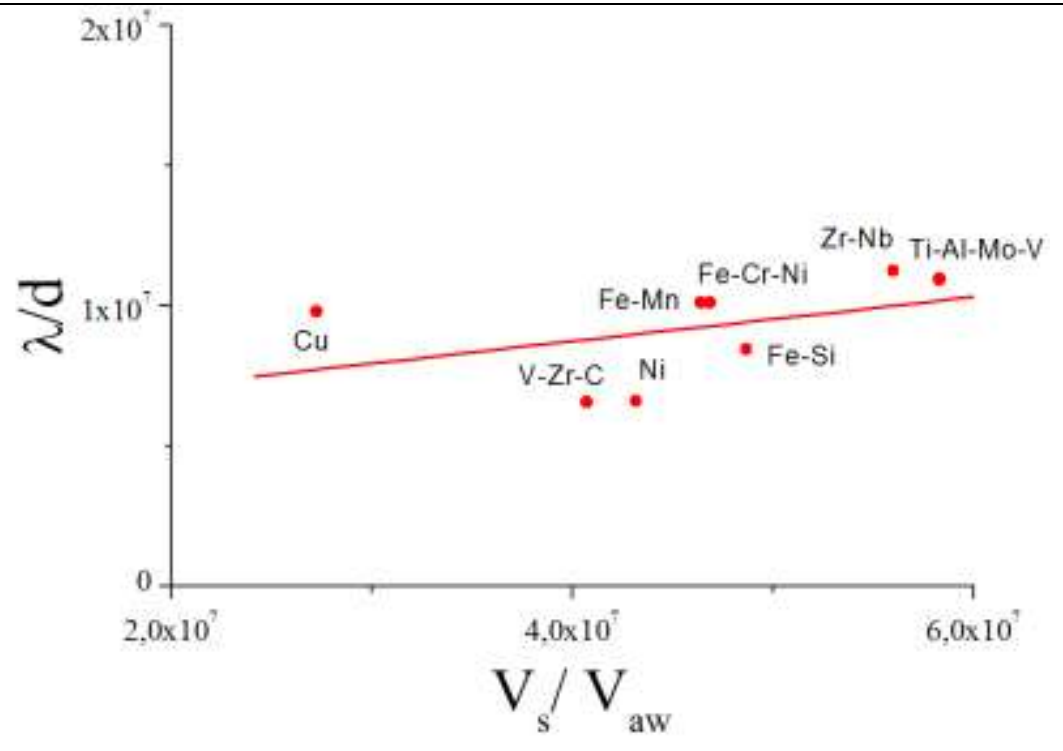

Figure 3. $\lambda / d$ ratio as a function of $V_{\perp} / V_{a w}$ ratio for metals and alloys investigated in this work (for exact compositions and crystal structures see Table I). Nearly linear dependence provides a verification of the validity of Eq. (1).

( $\lambda$ and $V_{a w}$ ) of localized plastic flow autowaves which are generated in deforming media on the other hand. In this case, it might be reasonable to regard the products $d \cdot V_{\perp}$ and $\lambda \cdot V_{a w}$ as invariants of elastic and plastic deformation processes, respectively. The above regularity suggests that the elastic and the plastic processes simultaneously involved in the deformation ( $\varepsilon<1$ and $\varepsilon \approx 1$, respectively) are closely related. The quantity $V_{\perp}$ is the rate of elastic stress redistribution in the deforming solid and the quantity $V_{a w}$ is the rate of localized plasticity front rearrangement in the same solid. Thus, the macro-scale localization of plastic deformation can no longer be regarded as a mere disturbance of plastic flow homogeneity by necking. Moreover, the localization phenomena are seen to be an attribute of the plastic deformation, with their characteristics being closely associated with and largely determined by the properties of crystal lattice.

\section{Conclusions}

In this work we investigated a possibility to determine macroscopic parameters for the description of localized plastic flow autowaves in deforming alloys from first-principles theoretical simulations. On the base of experimental data a significant regularity is established for the autowave process of plastic flow localization in a range of metals. Using experimentally determined autowave lengths $\lambda$ and propagation $\operatorname{rates} V_{a w}$, we 
establish by means of first-principles calculations a close correlation between the product of the macroscopic parameters of the autowave process, $\lambda \cdot V_{a w}$, and the product of the microscopic (lattice) parameters of material, $d \cdot V_{\perp}$, where $\mathrm{d}$ is the spacing between the

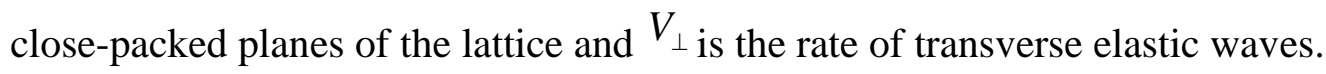

Considering the accuracy of theoretical calculations, we conclude that further improvement of the calculated parameters can be achieved by going beyond the DebyeGrüneisen theory of Ref. [24] and calculating the transverse elastic wave rate directly from ab initio elastic constants. Though this path is substantially more time consuming, it should lead to higher accuracy of the determination of microscopic parameters for the description of localized plastic flow auto-waves, especially in hexagonal and magnetic alloys.

\section{Acknowledgments}

This study was supported in part by the Ministry of Education and Science of the Russian Federation within the framework of Program "Research and Pedagogical Personnel for Innovative Russia (2009-2013)" (project no. 14.740.11.0037 of 01.09.2010) and within the framework of the program of development of "National research Tomsk state university (2010-2012)". The Russian Foundation for Basic Researches (Grant No. 1002-00-194 a, A.V.P. and Yu.Kh.V.) is acknowledged for financial support. Financial support from the Göran Gustafsson Foundation for Research in Natural Sciences and Medicine, the Swedish Foundation for Strategic Research (SSF) and the Swedish Research Council (VR) are gratefully acknowledged. Calculations have been performed at Swedish National Infrastructure for Computing (SNIC) and the Joint Supercomputer Center of RAS (Moscow).

\section{References.}

[1] L.B. Zuev, Ann. Phys., 10, 965-984 (2001)

[2] L.B. Zuev, Ann. Phys., 16, 286-310 (2007)

[3] R. J. McDonald, C. Efstathiou, P. Curath, J. Engng. Mat. Technol. 131, 692-703 (2009)

[4] A. Asharia, A. Beaudoin, R. Miller, Math. Mech. Solids, 13, 292-315 (2008)

[5] C. Fressengeas, A. Beaudoin, D. Entemeyer, Phys. Rev. B 79, 014108-1-014108-9 (2009)

[6] L.B. Zuev, V.V. Gorbatenko, S.N.Polyakov, Instrumentation for speckle interferometry and techniques for investigating deformation and fracture. Proceedings of SPIE, Vol. 4900, Part 2, pp. 1197-1208 (2002)

[7] H. Haken, Information and Self-Organization. A Macroscopic Approach to Complex Systems. Springer Verlag, Berlin (1989)

[8] L.B. Zuev, S.A. Barannikova, Natural Science 2. 476-483(2010)

[9] L.B. Zuev, S.A. Barannikova, J. Mod. Phys., 1, 1-8 (2010) 
[10] F.R.N. Nabarro, Z.S. Basinski, D.B. Holt, The Plasticity of Single Crystals. Tailor and Francis Ltd, London (1964)

[11] R. Hill, The Mathematical Theory of Plasticity. University Press, Oxford (1998)

[12] V.A. Davydov , V.S. Zykov, A.S. Michailov. Physics - Uspekhi, 161, 45-85 (1999)

[13] H. Kolsky, Stress waves in solid. Phoenix Edition, Dover (2003).

[14] X. H. Zhu, J. E. Carsley, W. W. Milligan and E. C. Aifantis, Scripta Mater. 36, 721-726 (1997).

[15] R.M. Martin: Electronic structure. Basic Theory and Practical Methods. Cambridge University Press, Cambridge (2004)

[16] A. V. Ruban and I. A. Abrikosov, Rep. Prog. Phys. 71, 046501 (2008).

[17] L. Vitos, I. A. Abrikosov, and B. Johansson, Phys. Rev. Lett. 87, 156401-4 pages (2001)

[18] A. V. Ponomareva, A.V. Ruban, N. Dubrovinskaya, L. Dubrovinskiy, and I. A. Abrikosov, Appl. Phys. Lett. 94, 181912 (2009), and references wherein.

[19] L. Dubrovinsky, N. Dubrovinskaia, F. Langenhorst, D. Dobson, D. Rubie, C.

Geshmann, I. A. Abrikosov, B. Johansson, V. I. Baykov, L. Vitos, T. Le Bihan, W. A.

Crichton, V. Dmitriev, and H.-P. Weber, Nature 422, 58 (2003), and references wherein

[20] I. A. Abrikosov, A. E. Kissavos, F. Liot, B. Alling, S. I. Simak, O. E. Peil, and A. V. Ruban, Phys. Rev. B 76, 014434 (2007).

[21] L. Dubrovinsky, N. Dubrovinskaia, O.Narygina, A. Kuznetzov, V. Prakapenka, L. Vitos, B. Johansson, A. S. Mikhaylushkin, S. I. Simak, and I. A. Abrikosov, Science 316, 1880 (2007)

[22] P. Olsson, I. A. Abrikosov, L. Vitos, and J. Wallenius, J. Nucl. Mat. 321, 84 (2003).

[23] L. Vitos, P. A. Korzhavyi, and B. Johansson, Phys. Rev. Lett. 88 (2002), 155501.

[24] V. L. Moruzzi, J. F. Janak, and K. Schwarz, Phys. Rev. B 37, 790 (1988).

[25] A. van de Walle and G. Ceder, Rev. Mod. Phys. 74, 11 (2002).

[26] I. A. Abrikosov, A. V. Ruban, D. Ya. Kats, and Yu. H. Vekilov, J. Phys.: Condens. Matter 5, 1271 (1993).

[27] L. Vitos, Phys. Rev. B 64, 014107 (2001).

[28] B. L. Györffy, et al., J. Phys. F: Met. Phys. 15 (1985), 1337.

[29] T. Gebhardt, D. Music, M. Ekholm, I. A. Abrikosov, J. von Appen, R. Dronskowski, D. Wagner, J. Mayer, and J. M. Schneider, Acta Materialia 59, 1493 (2011).

[30] A. K. Majumdar and P. v. Blanckenhagen, Phys. Rev. B 29, 4079, (1984) .

[31] B. Alling, T. Marten, and I. A. Abrikosov, Nature Materials 9, 283 (2010);

Phys. Rev. B 82, 184430 (2010). 